\title{
Effect of Intravenous Tranexamic Acid on Intracerebral Brain Hemorrhage in Traumatic Brain Injury
}

\author{
Hosein SAFARI, Parnian FARRAHI, Saleh RASRAS, Hosein Jafari MARANDI, Masoud ZEINALI \\ Ahvaz Jundishapur University of Medical Sciences, Department of Neurosurgery, Ahvaz, Islamic Republic of Iran
}

Corresponding author: Parnian FARRAHI pf81018ms@gmail.com

\section{ABSTRACT}

AIM: To determine the effect of Intravenous Tranexamic Acid (TXA) on traumatic intracerebral hemorrhage.

MATERIAL and METHODS: A total of 94 cases of TBI with intracerebral hemorrhage, admitted to the emergency department who did not need surgical intervention based on a primary brain spiral computed tomography (CT) scan, were randomly assigned into two groups of 47 patients. In the intervention group, intravenous TXA was administered as one gram of bolus and one gram every 6 hours for 48 hours, and in the control group, the placebo was administered in the same way. After 6,24 , and 48 hours all the cases underwent a brain CT scan. Scans were examined for the size and diameter of hematoma and the midline shift. The information regarding the level of consciousness, hematoma volume, and diameter on CT scan were recorded on arrival and 48 hours later.

RESULTS: Statistical results depict that while there was no considerable difference in the demographic aspect of the two groups, the volume and diameter of hematoma and the midline shift in the first CT scans and also their level of consciousness, the diameter and volume of hematoma and also the amount of hematoma expansion in follow up are significantly different in the two groups.

CONCLUSION: The present study showed that the prescription of TXA would reduce the amount of hematoma expansion in traumatic intracerebral hemorrhage, and that medication can be introduced to reduce morbidity and complications.

KEYWORDS: Traumatic Brain Injury, Intracerebral Hemorrhage, Tranexamic Acid

\section{INTRODUCTION}

$\mathrm{O}$ ne of the significant health issues worldwide is traumatic brain injury (TBI), according to the World Health Organization (WHO) statistics, and is one of the leading causes of death (9). The most common causes of TBI are vehicle collisions, falling from a height, beating injuries, sports injuries, and penetrating trauma. TBI is more common in people younger than 35 years of age with 2:1 Male to female ratio (14). TBI refers to the change in the function and pathology of the brain caused by an external force (13). TBI is caused by a direct impact to the head and neck or by a sudden motion of the head and neck. Head trauma can lead to a fracture in the skull, facial bones, and spinal vertebrae, a tear in the skin and other tissues, intracranial hemorrhage or diffuse axonal injuries (19). The most common complication associated with TBI is hemorrhage. If hematoma expansion can be prevented, not only will the morbidity rate decrease, but also the need for surgery (4). A brain inflicted with traumatic injuries will experience bleeding, inflammation, and edema. As a result, the intracranial pressure will increase. Subsequently, hypoxia, brain ischemia, and brain herniation are the irreparable damages that can lead to brain death (3). Intracerebral hematoma also includes brain contusion, which is a mixture of blood and natural tissue of the brain. Hemorrhage in either the white matter or the gray matter can lead to the expansion of the brain and mass effect, thus resulting in complications (21).
Hosein SAFARI (1): 0000-0003-3477-6344

Parnian FARRAHI (1) : 0000-0002-7066-5265

Saleh RASRAS (1) : 0000-0001-7605-7626
Hosein Jafari MARANDI (1) : 0000-0001-6420-9960

Masoud ZEINALI (10) : 0000-0002-3350-3275 
Surgical operations serve to save lives, relieve symptoms, and prevent complications. In cases of large hematoma, mass effect, impending brain herniation, pressure on the brain stem, or hydrocephalus, surgery is the top priority $(2,15)$. With respect to small hematomas or minor neurological deficits medication can be used as treatment. In recent decades there have been numerous interventional studies on medical treatment of brain damage (7). Tranexamic acid (TXA) is an antifibrinolytic agent used for the reduction of bleeding in different surgical operations. Previous studies have revealed that TXA decreases bleeding and lessens the need for blood transfusion without bringing about any serious side effects $(1,18)$. This article aimed to evaluate the effect of TXA on the fate of TBI-induced intracerebral hemorrhage.

\section{MATERIAL and METHODS}

The current study is a prospective, double-blind, randomized clinical trial in which patients with intracerebral hemorrhage, following TBI, who did not require surgical intervention, were enrolled. This study was approved by Ahvaz Jundishapur University of Medical Sciences Ethics Committee (IR. AJUMS. RES.1398.533). Informed consent was obtained from the patients or those accompanying the patient after explaining the procedure and its purpose.

Inclusion criteria in the current study were the presence of intracerebral hemorrhage, which did not indicate surgery in the initial computed tomography (CT) scan, and being between 16 to 65 years of age.

Exclusion criteria were patients with GCS of 3, patients requiring surgery for hematoma evacuation in the initial CT scan upon arrival, being under 16 or over 65 years of age, having macroscopic hematuria, history of anticoagulant medication, active vascular thrombosis or a history of it, hypercoagulative state, congenital or acquired hypercoagulopathy, history of vascular disease, valvular heart disease, renal failure, diabetes mellitus, being pregnant or breastfeeding, having a history of an allergic reaction to TXA, history of seizure or brain stroke, and patients' or relatives objection. The indications for a surgical operation following the assessment of the CT scans were intracerebral hemorrhage volume over $50 \mathrm{CC}$, intracerebral hemorrhage in frontal and temporal lobe with volume over 20 CC and GCS score 6-8 and midline shift of $5 \mathrm{~mm}$ or more or cisternal compression, progressive neurological deterioration referable to a lesion, medically refractory intracranial hypertension, or mass effect on CT scans.

The study was performed in the department of neurosurgery, Jundishapur University of Medical Sciences. Ninety-four cases of intracerebral hemorrhage following TBI who had been admitted to the Neurosurgery Emergency Ward of the hospital who did not meet exclusion criteria were randomly (using random number table) divided into two groups of 47 patients. Initially, the demographic information, including age, sex, and level of consciousness upon arrival, were recorded. The patients in the intervention group received the initial dosage of 1 gram TXA within the first 3 hours of admission, followed by 1 gram every 6 hours for 48 hours. In the control group, saline was administered as a placebo in the same manner; 6, 24, and
48 hours after starting injections. The patients in the 2 groups underwent a spiral brain CT scan and were evaluated for any change in the diameter and volume of the hematoma and also for any midline shift shown in the initial CT scans and the last CT scans. Furthermore, the patients were checked for their level of consciousness from the time they were admitted to when they were discharged.

Data are presented as mean \pm standard deviation for quantitative variables and frequency and percentage for qualitative variables. Data were analyzed using SPSS version 16.0 (SPSS, Chicago, IL) with Mann-Whitney and Chi-Square tests.

\section{RESULTS}

In the present study, in both control and intervention groups, the number of men was more than women, about 4-5 times more. Also, the average age of both groups was about 36 years, and was not significantly different between the intervention and control (Table I).

As can be seen in Table II, hematomas are measured in terms of width, length, height, and volume in both intervention and control groups. In the first CT scan, the results of the hematoma dimensions were similar in both groups and did not differ significantly in any of the width, length, height, and volume items. In the CT on Time 24, as is indicated in Table II, there is a significant increase of hematoma compared to the intervention group $(p<0.05)$ similar to the second CT results, and in the last CT scan, the hematoma was significantly lesser in length $(p=0.09)$, height $(p=0.04)$, and volume $(p=0.01)$ in the intervention group compared to the control. It was also observed in the control group, that the dimensions of the hematoma in the second and last CT scan had a notable increase in volume whereas this alteration did not occur in the intervention group. The dimension of the hematoma in the second CT did not change significantly compared to the last CT (Table II).

When evaluating hematoma growth rates between the first and last CT scans, it was noted that the mean volume change in the intervention group was significantly lower than in the control group; hematoma growth was $1.17 \pm 2.53 \mathrm{ml}$ in the intervention group and $6.85 \pm 11.77 \mathrm{ml}$ in the control group. This difference is statistically significant $(p=0.002)$. The occurrence of hematoma expansion was noted in 14 patients in the intervention group and 17 patients in the control group. This difference regarding the occurrence of hematoma expansion between the two groups was not statistically significant $(p=0.66)$.

Another item evaluated in the current experiment was midline shift in brain CT scan. An increase of less than $1 \mathrm{~mm}$ in midline shift can be seen between the first, second and last CT scan of both groups (this increase is higher in the control group), while this change is negligible and not significant. Also, betweengroups comparison of the midline shift in the first and next two CT scans was not significantly different (Table III).

In the intervention group, 1 case and in the control group 4 cases required surgical intervention based on follow up CT 
scans, but this difference was not statistically significant $(p=0.36)$.

Examination of patient consciousness based on GCS in both groups revealed an increase in scores of about 11 to 14 from admission to discharge. The level of consciousness was not significantly different between the two groups at the time of admission ( $p=0.91)$, after 24 hours $(p=0.53)$ and at the time of discharge $(p=0.49)$ (Table IV).

\section{DISCUSSION}

TXA is an antifibrinolytic agent used for the reduction of bleeding in different surgical operations. It serves as an antifibrinolytic by blockading the lysine binding sites of plasminogen molecules and preventing plasminogen transformation to plasmin. As a result, this prevented fibrin degradation and preserved the framework of fibrin matrix structure. Previous studies have revealed that TXA decreases bleeding and lessens the need for blood transfusion without

Table I: Demographic Information of the Intervention and Control Groups

\begin{tabular}{lccc}
\hline Groups & Intervention & Control & $\mathbf{p}$ \\
\hline Male & 40 & 38 & \\
\cline { 1 - 3 } Female & 7 & 9 & 0.78 \\
\hline Age & $36.2 \pm 15.1$ years & $36.4 \pm 14.1$ years & 0.94 \\
\hline
\end{tabular}

causing any severe side effects $(10,18)$. In cases of severe traumatic bleeding, early administration (within 3 hours) of TXA reduces the possibility of death by acute blood loss (5).

In this study, the effects of TXA on intracerebral hemorrhage due to TBI were analyzed. Demographic data, level of consciousness upon admission, hematoma diameters, volume, and midline shift in the first CT scans of the intervention and control groups showed no significant differences. While the occurrences of hematoma expansion and surgical intervention did not show any significant difference between the two groups, the hematoma diameters and volume in the second and last CT scans were significantly different between the two groups. The researchers in the present study have proved that TXA can reduce the amount of hematoma growth in traumatic intracerebral hemorrhage during 24 hours. No noteworthy differences in level of consciousness upon discharge, improvement in consciousness, and midline shift in the last CT scans were indicated regarding TXA consumption. Thus, although TXA can prevent an increase in the size of the hematoma, the clinical results do not show significant changes from admission to discharge.

Yutthakasemsunt et al. reported a 9\% reduction in progressive intracranial hemorrhage and also a reduction in death and unfavorable outcomes in patients with moderate to severe TBI who received TXA, but these changes were not statistically significant (22). Jokar et al., in a double-blind study, examined the effect of TXA in reducing hematoma expansion in patients with traumatic intracerebral hemorrhage. Forty-eight hours after admission, the mean increase in hematoma volume in

Table II: Hematoma Diameter Difference in the Initial, 24 Hours Later and Discharge in the Intervention and Control Groups

\begin{tabular}{lccccccccc}
\hline & \multicolumn{3}{c}{ First CT (Time 0) } & \multicolumn{3}{c}{ CT at 24 Hours } & \multicolumn{3}{c}{ CT at 48 Hours } \\
\hline Hematoma & Intervention & Control & $\mathbf{p}$ & Intervention & Control & $\mathbf{p}$ & Intervention & Control & $\mathbf{p}$ \\
\hline Width $(\mathrm{mm})$ & $15.1 \pm 7.3$ & $15.4 \pm 7.9$ & 0.83 & $16.3 \pm 2.3$ & $19.0 \pm 8.3$ & 0.09 & $16.3 \pm 7.8$ & $19.4 \pm 11.1$ & 0.11 \\
\hline Length $(\mathrm{mm})$ & $18.7 \pm 8.5$ & $19.3 \pm 9.3$ & 0.74 & $19.6 \pm 11.1$ & $23.6 \pm 12.3$ & $\mathbf{0 . 0 4}$ & $20.1 \pm 9.5$ & $24.9 \pm 13.3$ & $\mathbf{0 . 0 4}$ \\
\hline Height $(\mathrm{mm})$ & $22.3 \pm 11.3$ & $23.1 \pm 9.7$ & 0.71 & $23.4 \pm 8.8$ & $28.3 \pm 12.1$ & $\mathbf{0 . 0 4}$ & $23.4 \pm 11.8$ & $29 \pm 14.1$ & $\mathbf{0 . 0 4}$ \\
\hline Volume $(\mathrm{ml})$ & $5.0 \pm 6$ & $5.2 \pm 5.4$ & 0.85 & $6.0 \pm 9.3$ & $12.3 \pm 11.8$ & $\mathbf{0 . 0 1}$ & $6.2 \pm 7.4$ & $12.1 \pm 14.2$ & $\mathbf{0 . 0 1}$ \\
\hline
\end{tabular}

Table III: Midline Shift Difference Between the Intervention and Control Groups at the First, Second and Last CT Scan

\begin{tabular}{lccc}
\hline Groups & Intervention & Control & p \\
\hline Midline Shift at the Time 0 & $0.4 \pm 0.9 \mathrm{~mm}$ & $0.3 \pm 0.8 \mathrm{~mm}$ & 0.63 \\
\hline Midline Shift at the Time 24 & $0.6 \pm 1.0$ & $0.8 \pm 3.1$ & 0.62 \\
\hline Midline Shift at the Time 48 & $0.6 \pm 1.8 \mathrm{~mm}$ & $0.9 \pm 2.3 \mathrm{~mm}$ & 0.40 \\
\hline
\end{tabular}

Table IV: Level of Consciousness in the Intervention and Control Groups Upon Admission, 24 Hours Later and Discharge

\begin{tabular}{lccc}
\hline Groups & Intervention & Control & $\mathbf{p}$ \\
\hline GCS upon admission & $11.1 \pm 2.9$ & $11.1 \pm 3.0$ & 0.91 \\
\hline GCS upon Time 24 & $12.3 \pm 1.8$ & $11.1 \pm 2.6$ & 0.53 \\
\hline GCS upon discharge & $14.1 \pm 1.6$ & $13.9 \pm 1.9$ & 0.49 \\
\hline
\end{tabular}


Safari H. et al: Tranexamic Acid in Traumatic Brain Injury

the intervention group was significantly lower than the control group ( $p=0.04)$ (12). In a study by Perel et al., the effect of TXA on a hematoma in traumatic brain patients was investigated. This study showed that the use of TXA in the intervention group reduced the hematoma volume and mortality compared to the control group (16). In their study, the amount of hematoma in the intervention group was significantly lower than the control group, which is in line with the results of our study. Zehtabchi et al. in a systematic review, based on data collected from studies using TXA for traumatic brain hemorrhage, concluded that in the group treated with TXA, the amount of intracranial hemorrhage progression was lower than the group that did not receive TXA, but the improvement of clinical outcomes was statistically non-significant (23) which is consistent with the results of the present study. In a study by Fakharian et al. the effect of low doses of TXA on hematoma caused by TBI was evaluated, and it can be concluded that growth of posttraumatic hemorrhagic lesions did not show a significant difference between the two groups (8) which is inconsistent with the results of the present study. Tabesh et al., in a clinical trial study, examined the effect of TXA on various types of cerebral hemorrhage in brain trauma. In this study, they evaluated 190 patients with brain traumas and concluded that the effect of TXA infusion in patients with TBI was more dependent on the location and type of lesion, and its effects were more pronounced in patients with brain contusion (11). This result is consistent with the present study. The CRASH-3 trial reported that following $\mathrm{TBI}$, mortality and morbidity are significantly lower in patients receiving TXA. It was stated that the early administration of TXA (within 3 hours) could be more effective. Such methods are more effective in the treatment of mild and moderate cases of TBI rather than in severe cases (6). In a systematic review and meta-analysis, Weng et al. suggested that, in addition to the reduction of mortality rate and neurological complications, brain hematoma expansion in the recipients of TXA are significantly lower. The earlier the administration of TXA treatment, the smaller the size of hematoma will be (20). The aforementioned studies are in correlation with our study regarding hematoma growth. In the present study, even though the occurrence of expansion did not differ, the final hematoma volume and the amount of expansion were significantly lower in the TXA group.

In the present study, consciousness in the patients from both groups upon admission, after 24 hours and discharge was monitored. Data suggests there was no significant difference in the level of consciousness between the two groups at admission, after 24 hours and on discharge. Furthermore, after dividing patients into subgroups based on mild, moderate, and severe TBI (based on GCS upon admission), there was still no notable difference in the level of consciousness at admission and on discharge and improvement of consciousness. The importance of such categorization is because of the effectiveness of TXA in mild and moderate cases of a traumatic brain hemorrhage in studies reported recently (6).

Some studies state that TXA was effective in recovery of the level of consciousness $(6,20)$, which is contrary to our findings. This difference can be due to the sample size and follow-up time. Further studies are warranted to arrive at a conclusion. Furthermore, it should be noted that in this study, the level of consciousness upon discharge from the hospital is a dependent variable on the patient condition and time of discharge. There are pre-required levels of consciousness for patient discharge. Therefore, in a sense, it is not a perfect variable for reviewing the recovery of the level of consciousness. Besides, no follow up was done after discharge resulting in a short follow up time for assessing the level of consciousness. It is more practical to assess the levels of consciousness based on specific time intervals for a more extended period, even after discharge. Also, other types of morbidity, such as neurological deficits were neither documented in this study nor other studies in the literature.

The necessity for surgery presented itself for 4 cases in the control group and 1 case in the intervention group. Statistically speaking, such difference is minor, but from a different perspective, it could be suggested that TXA in TBI hemorrhage cases can help in the prevention of hematoma expansion, and as a result, the need for surgery is lessened. Further investigation in future studies with a larger sample size is recommended. Meanwhile, it should be noted that the midline shift has not been evaluated to date, and this marks a positive point of the present research, although the small sample size of the study can influence the conclusions. Thus the main limitation of the present study is the small sample size, which should be taken into consideration in future studies.

Previous studies have mostly focused on epidural hematoma and subdural hematoma, extensive contusions and looked at patients based on their GCS level, while the purpose of this paper was to focus on intraparenchymal hemorrhage including low and mild contusion, for the first time, taking into account the need for surgery and in the continuation of other studies. Also, in this study, we examined whether the above drug can reduce the need for surgery while other researchers have not mentioned this aspect.

\section{CONCLUSION}

This study suggests that although the intravenous TXA has no impact on the occurrence of hematoma expansion, it can lead to less volume expansion and, eventually, smaller hematoma volume. TXA has no significant effect on the level of consciousness, morbidity, and overall outcome. Further studies are needed to evaluate the effect of TXA on the final results of traumatic intracerebral hemorrhage.

\section{ACKNOWLEDGMENTS}

This study is a part of the specialty thesis of Parnian Farrahi and financially supported by the vice-chancellor of research affairs of the Ahvaz Jundishapur University of Medical Sciences.

The authors would like to thank of Golestan and Emam Hospital. 


\section{REFERENCES}

1. Ageron FX, Gayet-Ageron A, Ker K, Coats TJ, Shakur-Still H, Roberts I, Antifibrinolytics Trials Collaboration: The effect of tranexamic acid by baseline risk in acute bleeding patients: A meta-analysis of individual patient-level data from 28333 patients. Br J Anaesth 124(6):676-683, 2020

2. Algattas $\mathrm{H}$, Huang $\mathrm{JH}$ : Neurotrauma and repair research: Traumatic brain injury (TBI) and its treatments. Biomed Eng Comput Biol 5:51-56, 2013

3. Algattas $\mathrm{H}$, Huang $\mathrm{JH}$ : Traumatic brain injury pathophysiology and treatments: Early, intermediate, and late phases postinjury. Int J Mol Sci 15(1):309-341, 2013

4. Ciurli P, Formisano R, Bivona U, Cantagallo A, Angelelli P: Neuropsychiatric disorders in persons with severe traumatic brain injury. Journal of Head Trauma Rehabilitation 26:116126, 2010

5. Collaborators WT: Effect of early tranexamic acid administration on mortality, hysterectomy, and other morbidities in women with post-partum haemorrhage (WOMAN): An international, randomised, double-blind, placebo-controlled trial. Lancet 389(10084):2105-2116, 2017

6. CRASH-3 trial collaborators: Effects of tranexamic acid on death, disability, vascular occlusive events and other morbidities in patients with acute traumatic brain injury (CRASH-3): A randomised, placebo-controlled trial. Lancet 394(10210):1713-1723, 2019

7. Dewan MC, Rattani A, Gupta S, Baticulon RE, Hung YC, Punchak M, Agrawal A, Adeleye AO, Shrime MG, Rubiano AM, Rosenfeld JV, Park KB: Estimating the global incidence of traumatic brain injury. J Neurosurg 130:1-18, 2018

8. Fakharian $\mathrm{E}$, Abedzadeh M, Atoof F: Effect of tranexamic acid on prevention of hemorrhagic mass growth in patients with traumatic brain injury. World Neurosurgery 109:e748-e753, 2018

9. Faul M XL, Wald MM, Coronado VG: Traumatic Brain Injury in the United States: Emergency Department Visits, Hospitalizations and Deaths 2002-2006: Atlanta (GA) Centers for Disease Control and Prevention, National Center for Injury Prevention and Control, 2010

10. Gayet-Ageron A, Prieto-Merino D, Ker K, Shakur H, Ageron FX, Roberts I, Antifibrinolytic Trials Collaboration: Effect of treatment delay on the effectiveness and safety of antifibrinolytics in acute severe haemorrhage: A meta-analysis of individual patient-level data from 40138 bleeding patients. Lancet 391(10116):125-132, 2018

11. Homayoun T, Majid R, Sepideh S: The effect of tranexamic acid on traumatic brain hematomas. Journal of Isfahan Medical School (IUMS) 34(381):378-383, 2016
12. Jokar A, Ahmadi K, Salehi T, Sharif-Alhoseini M, RahimiMovaghar $\mathrm{V}$ : The effect of tranexamic acid in traumatic brain injury: A randomized controlled trial. Chin J Traumatol 20(1):49-51, 2017

13. Luauté J, Maucort-Boulch D, Tell L, Quelard F, Sarraf T, Iwaz $\mathrm{J}$, Boisson D, Fischer C: Long-term outcomes of chronic minimally conscious and vegetative states. Neurology 75(3):246, 2010

14. Miciak M, Gross DP, Joyce A: A review of the psychotherapeutic 'common factors' model and its application in physical therapy: The need to consider general effects in physical therapy practice. Scandinavian Journal of Caring Sciences 26(2):394-403, 2012

15. Murthy T, Bhatia P, Sandhu K, Prabhakar T, Gogna RL: Secondary brain injury: Prevention and intensive care management. Indian Journal of Neurotrauma 02(01):7-12, 2005

16. Perel P, Al-Shahi Salman R, Kawahara T, Morris Z, PrietoMerino D, Roberts I, Sandercock P, Shakur H, Wardlaw J: CRASH-2 (Clinical Randomisation of an Antifibrinolytic in Significant Haemorrhage) Intracranial Bleeding Study: The effect of tranexamic acid in traumatic brain injury - a nested, randomised, placebo-controlled trial. Health Technol Assess 16(13):iii-xii, 1-54, 2012

18. Roberts I, Belli A, Brenner A, Chaudhri R, Fawole B, Harris T, Jooma R, Mahmood A, Shokunbi T, Shakur H, CRASH-3 trial collaborators: Tranexamic acid for significant traumatic brain injury (The CRASH-3 trial): Statistical analysis plan for an international, randomised, double-blind, placebo-controlled trial. Wellcome Open Res 3:86, 2018

19. Traumatic Brain Injury in the United States: Fact Sheet [Internet]. CDC.gov. Atlanta (GA): CDC. [updated 2015 Jan 12].

20. Weng S, Wang W, Wei Q, Lan H, Su J, Xu Y: Effect of tranexamic acid in patients with traumatic brain injury: A systematic review and meta-analysis. World Neurosurgery 123:128-135, 2019

21. Wilkes S, McCormack E, Kenney K, Stephens B, Passo R, Harburg L, Silverman E, Moore C, Bogoslovsky T, Pham D, Diaz-Arrastia R: Evolution of traumatic parenchymal intracranial hematomas (ICHs): Comparison of hematoma and edema components. Front Neurol 9:527, 2018

22. Yutthakasemsunt $S$, Kittiwatanagul W, Piyavechvirat $P$, Thinkamrop B, Phuenpathom N, Lumbiganon P: Tranexamic acid for patients with traumatic brain injury: A randomized, double-blinded, placebo-controlled trial. BMC Emergency Medicine 13(1):20, 2013

23. Zehtabchi S, Abdel Baki SG, Falzon L, Nishijima DK: Tranexamic acid for traumatic brain injury: A systematic review and meta-analysis. Am J Emerg Med 32(12):1503-1509, 2014 